\title{
Visual Anosognosia (Anton-Babinski Syndrome): Report of Two Cases Associated with Ischemic Cerebrovascular Disease
}

\author{
Juan José Romero Carvajal ${ }^{{ }^{*}}$, Augusto Alejandro Arias Cárdenas ${ }^{1}$, Germán Zamora Pazmiño², \\ Patricio Abad Herrera ${ }^{3}$ \\ ${ }^{1}$ Resident of Internal Medicine Department, Hospital Metropolitano, Universidad \\ Internacional del Ecuador, Quito, Ecuador \\ ${ }^{2}$ Department of Neuroradiology, Hospital Metropolitano, Quito, Ecuador \\ ${ }^{3}$ Department of Neurology, Hospital Metropolitano, Quito, Ecuador \\ Email: "homero40@hotmail.com
}

Received May 28, 2012; revised June 23, 2012; accepted June 30, 2012

\begin{abstract}
Visual anosognosia or Anton-Babinski syndrome is a rare neurological condition related to cortical blindness. The patients deny their blindness and affirm adamantly that they are capable of seeing. The clinical presentation includes confabulations and sometimes confusional states. In this article we report two patients with anosognosia related to ischemic stroke in two different sets of etiology and pathogenesis. We describe the major clinical manifestations of this syndrome and review the current medical literature. Two patients were identified, a 96-year-old male with visual anosognosia secondary to a right posterior cerebral artery thrombosis, and a 56-year-old female with the same syndrome but related to central nervous system angiitis in relation with multiple sclerosis and Hashimoto's thyroiditis. Visual anosognosia or Anton-Babinski syndrome is a rare neurological condition. However the ischemic vascular cerebral disease is a frequent etiology. We believe that this is the first report of this syndrome in relation to angiitis with a clear autoimmune pathogenesis.
\end{abstract}

Keywords: Visual Anosognosia; Cortical Blindness; Anton-Babinski Syndrome; Stroke; MRI

\section{Introduction}

Cortical blindness matches several clinical criteria [1]: loss of all visual sensations, loss of menace reflex, preservation of pupillary reflexes, a normal fundoscopic examination and preservation of ocular movements. Visual anosognosia, or Anton-Babinski syndrome is a rare complication of cortical blindness, where the patients deny their visual deficit [2]. Damage of the visual association cortex has been thought as one of the main causes explaining the loss of awareness of the visual deficit [3], along with damage of other pathways connecting the visual cortex with the systems that process the information received from the senses. Ischemic cerebrovascular disease causing cortical blindness is the most common etiology of this syndrome [4].

We herein describe two cases and a review of the literature about the visual anosognosia.

"Corresponding author.

\section{Case Presentation}

\subsection{Patient \#1}

A 96-year-old man was admitted to Emergency Room with severe headache and sudden loss of vision. He had a cutaneous carcinoma resected about three years ago, and he was currently on treatment for hypertension.

On admission he was awake and oriented. Blood pressure was $180 / 100$, and he had a mild paresia on his left arm. Ophthalmologic exam confirmed a severe vision loss, ocular movements, as well as photo motor reflex, were preserved. Fundoscopic examination revealed changes secondary to chronic hypertension. The patient sustained that he was able to see, despite the objective evidence of vision loss.

The diffusion images on the brain MRI of the brain demonstrated ischemic areas on the occipital lobes, specially on the right side (Figure 1). A brain CT angiography showed a stenotic segment on the posterior right cerebral artery (Figure 2). 

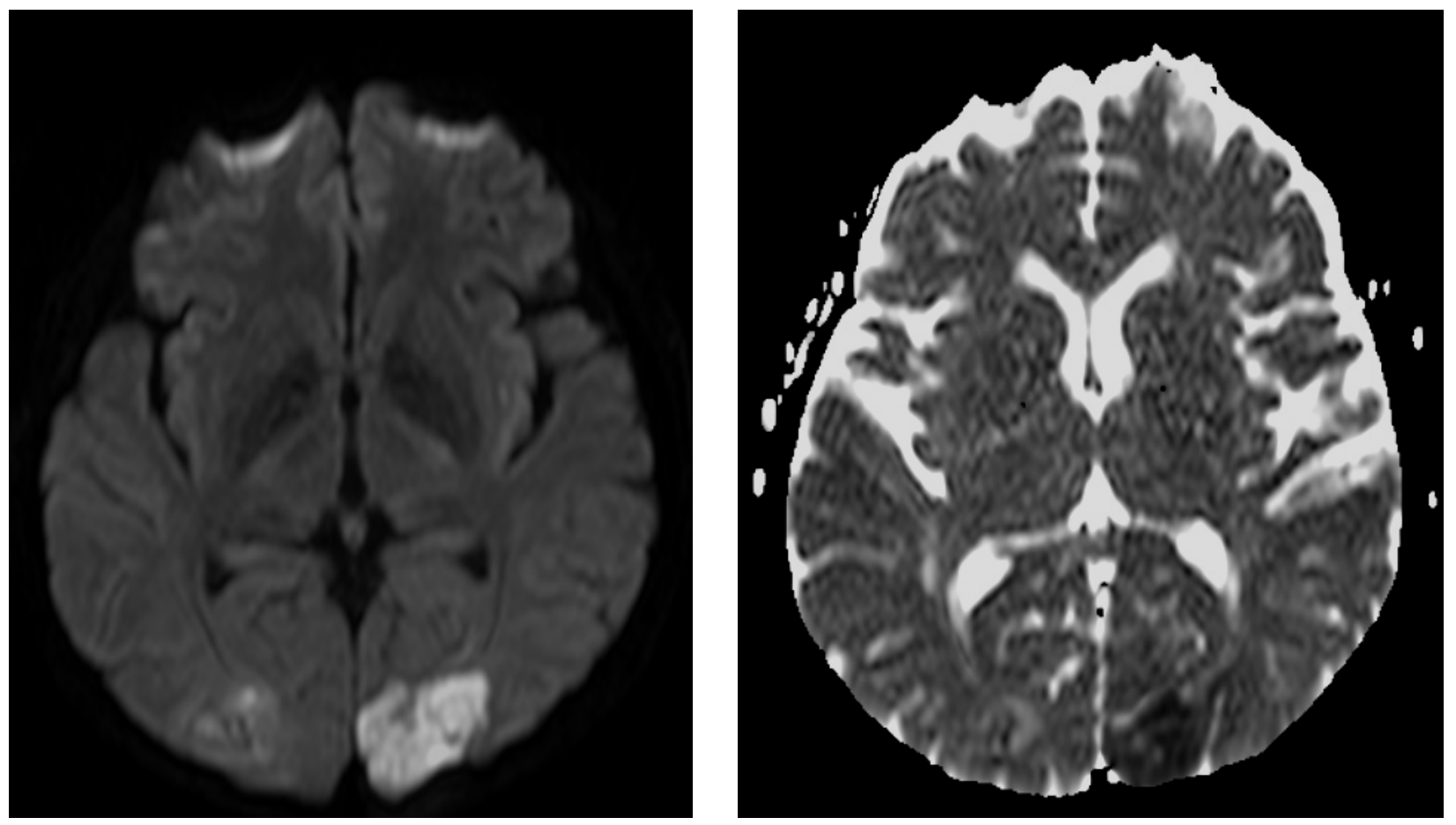

Figure 1. Diffusion images on cranial MRI showed restricted diffusion of water on the occipital lobes, findings suggestive of an ischemic lesion.

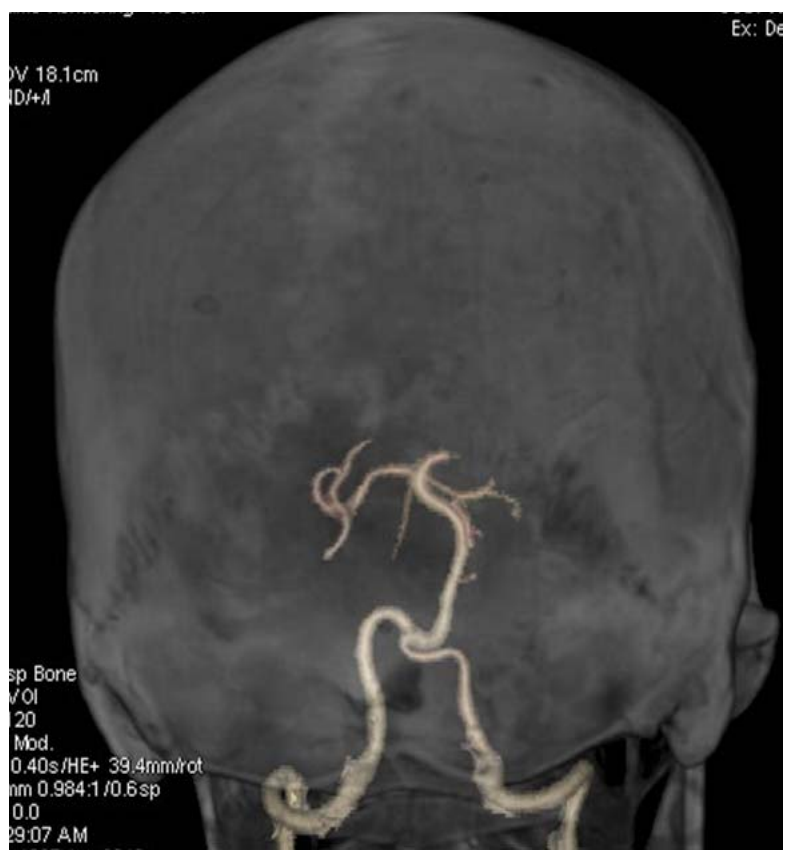

(a)

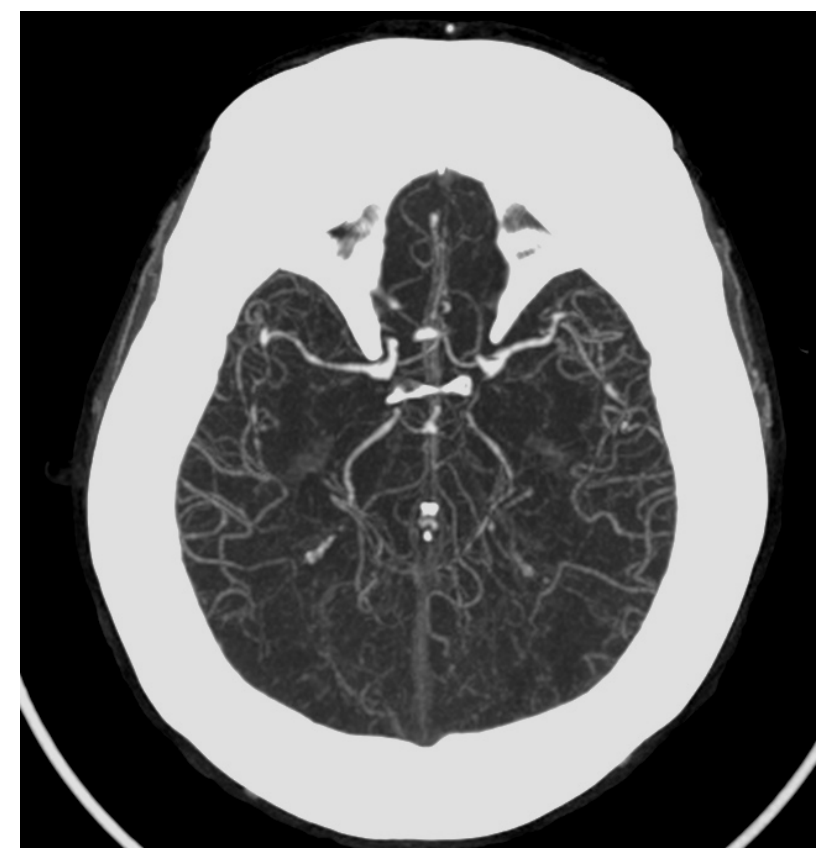

(b)

Figure 2. (a) Volumetric reconstruction and (b) maximum intensity projection of cerebral angiotomography of Patient 1, showing irregularity and a bilateral decrease of caliber of the posterior cerebral arteries.

During hospitalization the patient denied his visual deficit. He would fabulate about the landscape across the window on a room with no windows, and made wrong statements about the physician's tie (for example, referring to the tie's color when the doctor wasn't wearing one).

Visual loss remained stable during hospitalization, high blood pressure was controlled. The patient was discharged with secondary prevention treatment for stroke. 


\subsection{Patient \#2}

A 56-year-old woman with history of Hashimoto's thyroiditis and multiple sclerosis currently on interferon beta-1a was admitted to the Emergency Room with confusional state and significant psychomotor agitation that required sedation and ventilator support. Acute encephalitis was suspected, but cerebrospinal fluid studies, as well as herpes serology were negative.

Once sedation was discontinued and ventilatory support was retired, it became evident that the patient was not capable of identifying objects, neither perceiving light changes. Ocular movements and pupil reflexes were present. Fundoscopic examination was normal. Thyroid studies revealed suppressed TSH $(0.006 \mathrm{uU} / \mathrm{mL})$ and high titers of antithyroid antibodies $(79.83 \mathrm{UI} / \mathrm{mL})$. Thyroxine levels were $1.34 \mathrm{ng} / \mathrm{dL}$. Erythrocyte sedimentation rate was high also $(65 \mathrm{~mm} / 2 \mathrm{~h})$

MR images demonstrated acute ischemic lesions with total compromise of left occipital lobe (Figure 3). CT angiogram brain scan revealed a diffuse and moderate narrowing of brain arteries, suggestive of vasculitis (Figure 4).

During hospitalization, patient was not aware of her visual deficit. She would mistakenly describe the color of her husband's suit, or she would take the newspaper and pretended she was reading it.

The patient was put on methylprednisolone boluses, and later she received intravenous cyclophosphamide, with partial recovery of her deficit.

\section{Review of the Literature}

Cortical blindness matches the following clinical criteria [1]: a) loss of all visual sensations, including the perception of light and dark; b) loss of menace reflex; c) preservation of light and accommodation pupillary reflexes; d) a normal fundoscopic examination, and e) preservation of ocular movements.

Visual anosognosia, or Anton-Babinski syndrome, is a rare complication of cortical blindness, where the patients deny their visual deficit [2]. These patients also have damage on visual association cortex, causing the loss of the concept of vision and the awareness of their deficit [3,5].

Theories that try to explain the unawareness of deficit on the Anton-Babinsky syndrome describe disconnection phenomena. The first theory describes a conscious awareness system (CAS) located on the parietal lobes, which monitors all the information received from the senses. This system connects with other, located on the frontal lobes, which integrates the information, in order to perform complex cognitive tasks. In Anton-Babinski syndrome, damage of association pathways between visual cortex and CAS would be responsible for the lack of awareness of the visual deficit [6]. Furthermore, the disconnection of the visual areas from other, such as language areas, makes the patients unable to describe the visual stimuli, and, because of that, fabulate an answer.
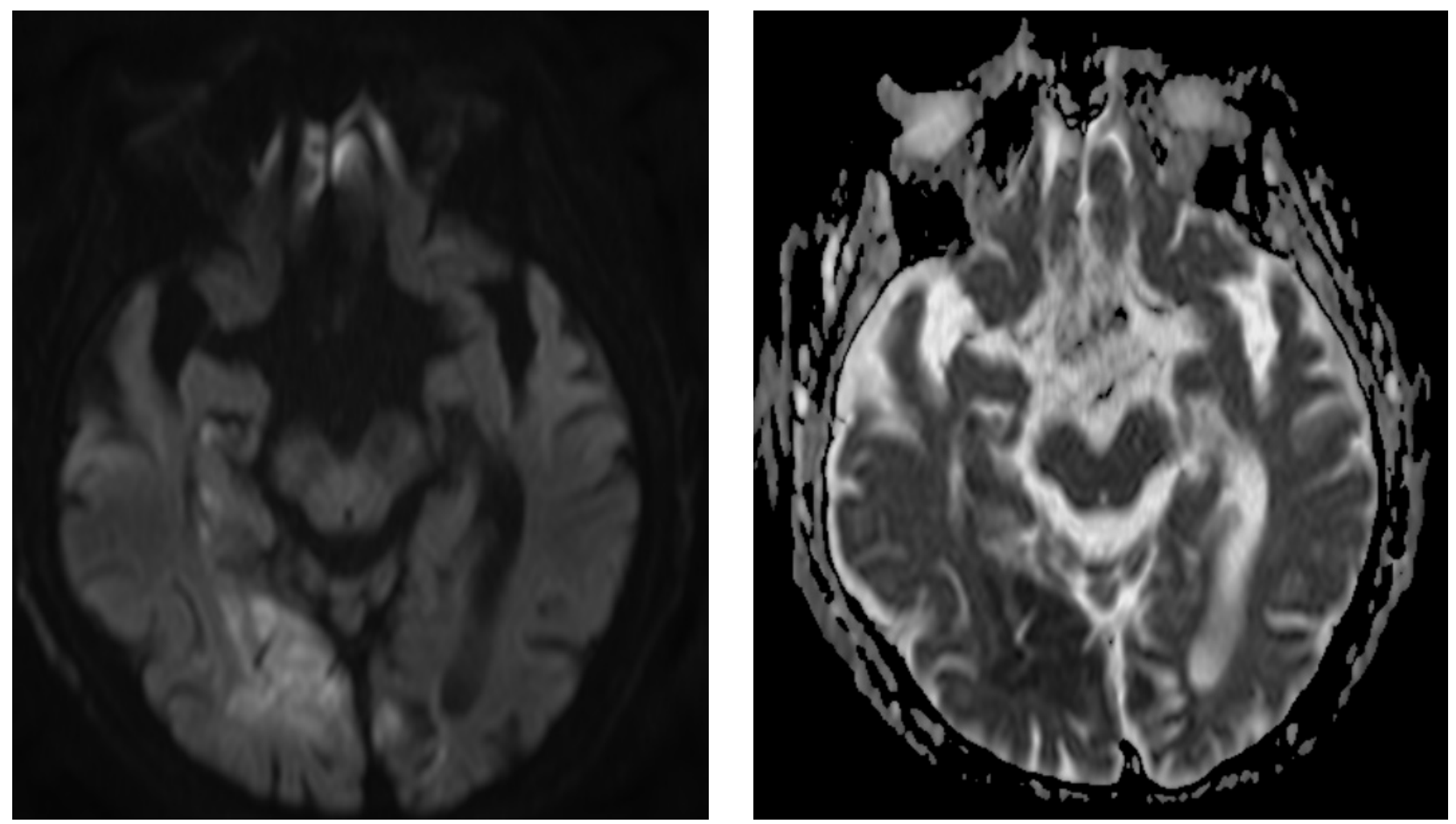

Figure 3. Diffusion images on cerebral MRI of Patient 2, demonstrating restricted diffusion of water on occipital lobes. 


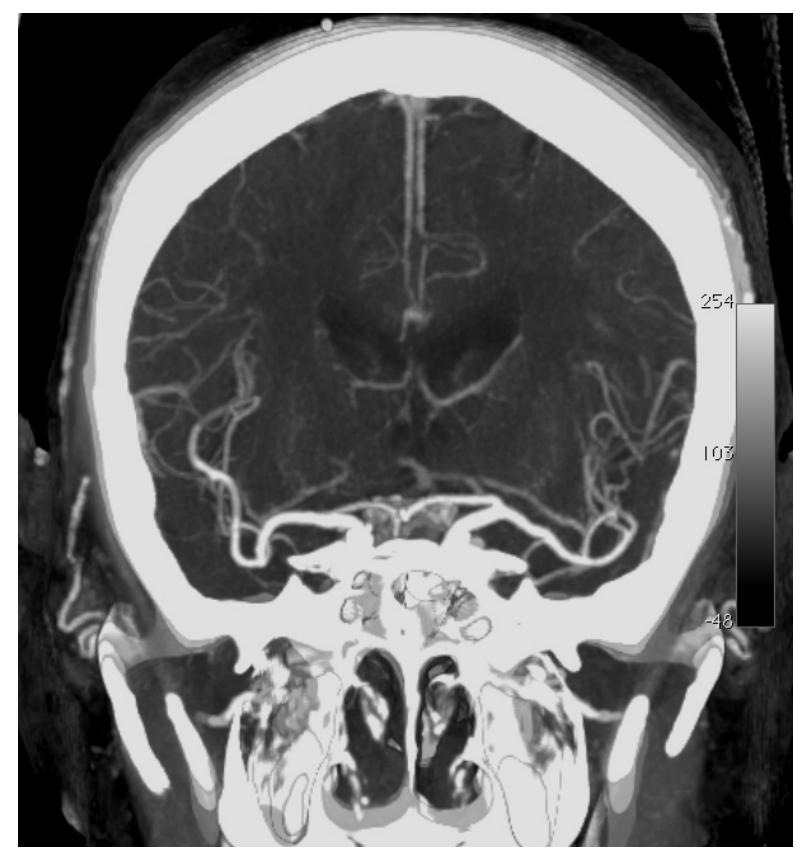

(a)

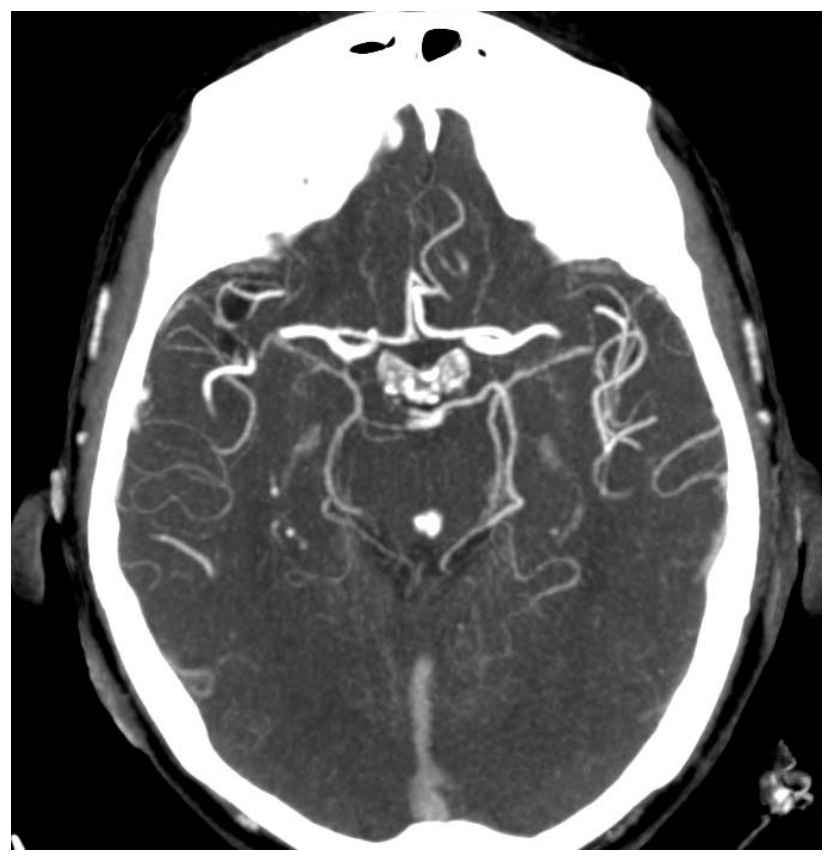

(b)

Figure 4. Maximum intensity projections (a) coronal and (b) axial of CT angiography of Patient 2, showing a decrease of the caliber of the posterior cerebral arteries.

In addition the disconnection phenomena, other neuropsychologic mechanisms have been described, such as the signal transmission to the visual monitor (located on the visual association cortex) from a secondary visual system, located on the superior colliculus, pulvinar and temporoparietal regions [5]. In the absence of transmission on the geniculocalcarine pathway, this secondary visual pathway would allow blind patients to fabulate.

Ischemic cerebrovascular disease, as a cause of cortical blindness, is the most common etiology of AntonBabinski syndrome [4,7]. Other diseases described as causes are MELAS [8], preeclampsia [2], obstetric hemorrhage [9], trauma [10], adrenloeucodistrophy [11], hypertensive encephalopathy [12] and angiographic procedures [13].

The first description of visual anosognosia was made by Renaissance French writer Michel de Montaigne (1533-1592). In the second book of his Essais, near the final of the twelfth chapter, he describes a nobleman who denied his own blindness [14]. In 1895, the Austrian psychiatrist and neurologist Gabriel Anton (1858-1933) described the case of Juliane Hochriehser, a 69-year-old dairymaid who had anosognosia with cortical deafness due to a lesion on her both temporal lobes [15]. Gabriel Anton described other cases of patients with objective blindness and deafness who denied their deficits. In 1914 the French-Polish neurologist Joseph François Babinski (1857-1932) used for the first time the term "anosognosia" to describe the unawareness of the deficit in pa- tients with hemiplegia [16].

We haven't found cases of visual anosognosia related to angeitis of the central nervous system [17]. Our patient had a history of relapsing-remitting multiple sclerosis and Hashimoto's thyroiditis. The laboratory results were remarkable for a high ESR and suppressed values of TSH. The MRI study showed a diffuse involvement of small and medium vessels of the central nervous system. Our patient also had lower right limb anosognosia and leftright confusion, probably related with CAS (conscious awareness system).

\section{Conclusion}

Visual anosognosia, or Anton-Babinski syndrome is a rare neurological condition, with well defined clinical criteria. Neuroimaging studies, along with a complete clinical evaluation, are of great value for the prognosis of the patients. We think our case related with central nervous system vasculitis is one of the first described in literature. Identifying the cause of visual anosognosia is important in establishing de prognosis of the patients.

\section{REFERENCES}

[1] G. Celesia and G. Brigell, "Cortical Blindness and Visual Anosognosia," In: Disorders of Visual Processing: Handbook of Clinical Neurophysiology, Elsevier, Edinburgh, 2005, pp. 429-440.

[2] M. Misra, S. Rath and A. B. Mohanty, "Anton Syndrome 
and Cortical Blindness Due to Bilateral Occipital Infarction," Indian Journal of Ophthalmology, Vol. 37, No. 4, 1989, p. 196.

[3] G. Goldenberg, W. Mullbacher and A. Nowak, "Imagery without Perception-A Case Study of Anosognosia for Cortical Blindness," Neuropsychologia, Vol. 33, No. 11, 1995, pp. 1373-1382. doi:10.1016/0028-3932(95)00070-J

[4] M. Maddula, S. Lutton and B. Keegan, "Anton's Syndrome Due to Cerebrovascular Disease: A Case Report," Journal of Medical Case Reports, Vol. 3, 2009, p. 9028. doi:10.4076/1752-1947-3-9028

[5] K. M. Heilman, "Anosognosia: Possible Neuropsychological Mechanisms," In: P. George, G. P. Prigatano and D. L. Schacter, Eds., Awareness of Deficit after Brain Injury: Clinical and Theoretical Issues, Oxford University Press, New York, 1991, pp. 53-62.

[6] S. M. McGlynn and D. L. Schacter, "Unawareness of Deficits in Neuropsychological Syndromes," Journal of Clinical and Experimental Neuropsychology, Vol. 11, No. 2, 1989, pp. 143-205. doi:10.1080/01688638908400882

[7] M. S. Aldrich, A. G. Alessi, R. W. Beck and S. Gilman, "Cortical Blindness: Etiology, Diagnosis, and Prognosis," Annals of Neurology, Vol. 21, No. 2, 1987, pp. 149-158. doi:10.1002/ana.410210207

[8] M. Alemdar, P. Iseri, M. Selekler, et al., "MELAS Presented with Status Epilepticus and Anton-Babinski Syndrome; Value of ADC Mapping in MELAS," Journal of Neuropsychiatry \& Clinical Neurosciences, Vol. 19, No. 4, 2007, pp. 482-483. doi:10.1176/appi.neuropsych.19.4.482

[9] P. A. Argenta and M. A. Morgan, "Cortical Blindness and Anton Syndrome in a Patient with Obstetric Haemorrhage," Obstetrics \& Gynecology, Vol. 91, No. 5, 1998, pp. 810-812. doi:10.1016/S0029-7844(97)00718-7

[10] K. D. McDaniel and L. D. McDaniel, “Anton's Syndrome in a Patient with Posttraumatic Optic Neuropathy and Bifrontal Contusions," Archives of Neurology, Vol. 48, No. 1, 1991, pp. 101-105. doi:10.1001/archneur.1991.00530130113028

[11] R. Trifiletti, E. Syed, C. Hayes-Rosen, E. Parano and P. Pavone, "Anton-Babinski Syndrome in a Child with EarlyStage Adrenoleukodystrophy," European Journal of Neurology, Vol. 14, No. 2, 2007, pp. e11-e12. doi:10.1111/j.1468-1331.2006.01581.x

[12] J. Nguyen-Lam and M. C. Kiernan, "Acute Cortical Blindness Due to Posterior Reversible Encephalopathy," Journal of Clinical Neuroscience, Vol. 15, No. 10, 2008, pp. 1182-1185. doi:10.1016/j.jocn.2007.04.025

[13] B. N. Alp, N. Bozbuğa, M. A. Tuncer and C. Yakut, "Transient Cortical Blindness after Coronary Angiography," Journal of International Medical Research, Vol. 37, No. 4, 2009, pp. 1246-1251.

[14] R. Bernoulli, "Michel de Montaigne (1533-1592): Report of a Case of Not Perceiving Own Blindness," Gesnerus, Vol. 47, No. 1, 1990, pp. 13-20.

[15] E. Kumbier, K. Haack and S. C. Herpertz, "The Juliane Hochriehser Case-A Classical Case of Anosognosia," Wurzbg Medizinhist Mitt, Vol. 26, 2007, pp. 53-74.

[16] K. Langer, "Babinski's Anosognosia for Hemiplegia in Early Twentieth-Century French Neurology," Journal of the History of the Neurosciences, Vol. 18, No. 4, 2009, pp. 387-405. doi:10.1080/09647040802537064

[17] P. M. Moore, "Neurological Manifestations of Vasculitis; Update on Immune Pathogenic Mechanisms and Clinical Features," Annals of Neurology, Vol. 37, No. 1, 1995, pp. 131-141. doi:10.1002/ana.410370713 